\title{
CARACTERIZAÇÃO E DEMANDA DE UM SERVIÇO DE ATENDIMENTO PSICOLÓGICO ON-LINE NO CONTEXTO DA PANDEMIA DE COVID-19
}

DOI: 10.22289/2446-922X.V7N1A7

\author{
Antonio Augusto Pinto Junior ${ }^{1}$ \\ Maristela Siqueira Macedo de Paula Santos \\ Teresa Cristina Rangel Credidio Zampieri
}

\section{RESUMO}

A pandemia da COVID-19 tem sido considerada a mais grave e importante crise de saúde pública neste momento. Além das taxas alarmantes de morbimortalidade no mundo inteiro, essa doença vem desencadeando vários tipos de sofrimento psíquico, em função do isolamento social, medo de adoecer ou de contaminar amigos ou familiares. Em função disso, torna-se necessário o desenvolvimento de serviços de tratamento psicológico para aqueles que apresentam alguma forma de transtorno mental decorrente dessa pandemia e seus efeitos. O presente trabalho descreve a experiência e os resultados de um serviço de atendimento psicológico on-line desenvolvido pela Secretaria Municipal de Saúde de Guaratinguetá/SP que objetivou oferecer um espaço de escuta para a população geral e profissionais de saúde visando minorar o sofrimento e favorecer uma melhor qualidade de vida. Os resultados mostram que foram atendidos de forma remota 145 pessoas, predominantemente do sexo feminino (79,3\%), na faixa etária entre 41-59 anos (35,2\%) e entre $60-80$ anos (22,1\%). Grande parte da população atendida apresenta ensino médio completo $(38,0 \%)$, é dona de casa (26,9\%) ou está desempregada (16,6\%). Quanto a queixa, observou-se que a grande maioria referiu quadros de ansiedade (45,4\%) ou de depressão (18,9\%). A partir desses dados, conclui-se que são necessárias a continuidade e a expansão desse tipo de serviço para a proteção e a promoção da saúde mental da população em geral e, mais especificamente, dos profissionais de saúde que estão na linha de frente de combate à pandemia da COVID-19.

Palavras-chave: COVID-19; Psicoterapia; Assistência à Saúde Mental.

\section{CHARACTERIZATION AND DEMAND OF AN ON-LINE PSYCHOLOGICAL SERVICE IN THE CONTEXT OF THE COVID- 19 PANDEMIC}

\section{ABSTRACT}

The COVID-19 pandemic has been considered the most serious and important public health crisis at this time. In addition to the alarming rates of morbidity and mortality worldwide, this disease has been triggering various types of psychological distress, due to social isolation, fear of falling ill or

\footnotetext{
${ }^{1}$ Endereço eletrônico de contato: antonioaugusto@id.uff.br

Recebido em 30/12/2020. Aprovado pelo conselho editorial para publicação em 08/02/2021.
} 
contaminating friends or family. Therefore, it is necessary to develop psychological treatment services for those who have some form of mental disorder resulting from this pandemic and its effects. This paper describes the experience and results of an online psychological care service developed by the Municipal Department of Health in Guaratinguetá, in the state of Sao Paulot, hat aimed to provide a psychological treatment to the general population and health professionals in order to alleviate suffering and favor a better quality of life. The results show that 145 people were treated remotely, predominantly female $(79.3 \%)$, aged between $41-59$ years $(35.2 \%)$ and between $60-80$ years $(22.1 \%)$. A large part of the population treated has completed high school $(38.0 \%)$, is a housewife $(26.9 \%)$ or is unemployed $(16.6 \%)$. About the complaint, it was observed that the vast majority referred to anxiety $(45.4 \%)$ or depression (18.9\%). From these data, it is concluded that the continuity and expansion of this type of service is necessary for protection and promotion of the mental health of the population in general and, more specifically, of the health professionals who are on the front line of combat to the COVID-19 pandemic.

Keywords: COVID-19; Psychotherapy; Mental Health Assistance.

\section{CARACTERIZACIÓN Y DEMANDA DE UN SERVICIO PSICOLÓGICO ON-LINE EN EL CONTEXTO DE LA PANDEMIA DEL COVID-19}

\section{RESUMEN}

La pandemia de COVID-19 es considerada la crisis de salud pública más grave en este momento. Además de las alarmantes tasas de morbilidad/mortalidad en todo el mundo, esta enfermedad viene desencadenando diversos tipos de malestar psicológico, debido al aislamiento social, miedo a enfermar o contaminar a amigos o familiares. Asi, es necesario desarrollar servicios de tratamiento psicológico para quienes padecen algún tipo de trastorno mental resultante de esta pandemia y sus efectos. Este trabajo describe la experiencia y resultados de un servicio de atención psicológica online desarrollado por la Secretaría Municipal de Salud de Guaratinguetá, en el estado de São Paulo, que tuvo como objetivo ofrecer un espacio de escucha a la población en general y a los profesionales de la salud con el fin de aliviar el sufrimiento y favorecer un mejor calidad de vida. Los resultados muestran que 145 personas fueron tratadas online, predominantemente mujeres $(79,3 \%)$, con edades entre $41-59$ años $(35,2 \%)$ y entre $60-80$ años $(22,1 \%)$. Una gran parte de la población atendida tiene estudios secundarios completos $(38,0 \%)$, es ama de casa $(26,9 \%)$ o está desempleada (16,6\%). En cuanto a la queja, se observó que la gran mayoría se refirió a ansiedad $(45,4 \%)$ o depresión (18,9\%). Asi, se concluye que la continuidad y expansión de este tipo de servicio es necesaria para la protección y promoción de la salud mental de la población y, más específicamente, de los profesionales de la salud que se encuentran en la primera línea de combate a la pandemia de COVID-19.

Palabras clave: COVID-19; Psicoterapia; Atención a la Salud Mental.

\section{INTRODUÇÃO}

A pandemia da COVID-19, causada pelo novo Coronavírus (SARS-CoV-2), revela-se como a mais grave e importante crise de saúde pública que ameaça a humanidade neste momento. Até o final de dezembro de 2020, a Organização Mundial da Saúde (OMS) registrou mais de 80.000.000 e quase 2.000.000 de mortes no planeta, cujos números não param de subir (WHO, 2020). O Brasil, 
até esse período, já contabilizou quase 7.500 .000 casos, com mais de190.000 óbitos, ocupando a segunda posição entre os países em relação ao número de mortes (Ministério da Saúde, 2020), valor acima de todas as nações em desenvolvimento.

Diante desse quadro e da ausência, ainda, de um plano eficaz de imunização, especialmente no Brasil2, as estratégias de distanciamento social e o uso de dos Equipamentos de Proteção Individual (EPI) têm sido apontados como a mais importante forma de intervenção para o controle da COVID-19. Contudo, as mudanças implementadas por esse novo modo de vida, além de gerar uma quebra da rotina das pessoas, passa a desencadear muitas incertezas, comprometendo a forma como os indivíduos administram o seu cotidiano, a capacidade de domínio emocional e também de competência social (Dugnani, 2020; Ornell, 2020; Dal'Bosco, 2020).

A OMS (WHO, 2002) já, há muito, considera que a saúde mental e a saúde física são inseparáveis e que uma influencia a outra. Além disso, os estados afetivos angustiados e deprimidos desencadeiam mudanças no funcionamento endócrino e imunológico criando suscetibilidades para outras doenças físicas. Em uma pandemia, como essa, o medo pode aumentar os níveis de ansiedade e estresse em indivíduos saudáveis e intensifica os sintomas daqueles com transtornos psicológicos preexistentes (Dal'Bosco, 2020).

Assim, a pandemia da COVID-19 tende a tornar o sofrimento psíquico mais agudo. Os recursos de como lidar com a angústia podem ficar mais limitados, como passear, viajar, estabelecer novos vínculos afetivos e de trabalho, gerando sentimentos de impotência e inadequação. Tudo isso fica mais sério com a transformação do sofrimento em sintomas. Da mesma forma, a elaboração do luto por perda de amigos ou familiares é mais dolorosa, com efeitos póstraumáticos intensificados (Schmidt, 2020; Ornell, 2020; Dugnani, 2020).

Deve-se pontuar que é importante as pessoas ficarem atentas aos sintomas persistentes de angústia, tristeza, medo, conflitos que se manifestem de forma intensa ou que provoquem dificuldades profundas no cotidiano. Nesses casos, é importante procurar ajuda profissional. A partir de estratégias de cuidado especializado, o profissional de psicologia pode ajudar a prevenir complicações, começando por reconhecer e acolher receios e medos, manter ativa a rede sócio afetiva, considerando os protocolos de segurança que o atual momento exige. Com esse tipo de ajuda especializada, pode-se entender que novos comportamentos adaptativos são necessários para atender as demandas da nova realidade que se impõe (Schmidt, 2020; Dal'Bosco, 2020; Ornell, 2020).

Em se tratando, especificamente, dos profissionais de saúde que, muitas vezes, estão na linha de frente no enfrentamento dessa doença, Teixeira et al (2020), afirmam que, além do risco

\footnotetext{
${ }^{2}$ Até o momento da redação desse artigo, embora já havia algumas vacinas aprovadas ao redor do mundo, e países já iniciando a imunização de sua população, o Brasil, ainda, não tinha definido com rigor seu plano de vanização contra a COVID-19.
}

Rev. Psicol Saúde e Debate. Fev., 2021:7(1): 94-106. 
de contaminação que pode gerar afastamento do trabalho, doença e morte, a atuação junto aos pacientes com teste positivo para COVID-19 faz com que muitos desenvolvam intenso sofrimento psíquico, com quadros de ansiedade generalizada, distúrbios do sono, medo de adoecer, morrer ou de contaminar colegas e familiares. Para enfrentar essa situação, os autores destacam a importância de se desenvolver estratégias de capacitação de pessoal, atendimento psicológico especializado e a reorganização do processo de trabalho na atenção básica para o enfrentamento da pandemia, visando às medidas necessárias para a proteção e a promoção da saúde física e mental dos profissionais e trabalhadores da saúde.

Em função do exposto, e visando cuidar da saúde mental da população de durante a pandemia da COVID-19, a Secretaria Municipal de Saúde de Guaratinguetá/SP desenvolveu, em parceria com psicólogos voluntários e servidores municipais, um programa de atendimento psicológico on-line para pacientes que foram testados positivo para essa doença, ou em isolamento social, além de profissionais da saúde que estão na linha de frente dos atendimentos de pacientes com sintomas da doença. Deve-se salientar que Guaratinguetá é um município de médio porte, do interior do estado de São Paulo, localizado na região do Vale do Paraíba, e com uma população total de 121.073 habitantes (IBGE, 2017).

A prestação de serviços psicológicos por Tecnologias da Informação e da Comunicação (TICs), também conhecidos como atendimentos psicológicos on-line, de acordo com Viana (2020), é um tipo de serviço regulamentado pelo Conselho Federal de Psicologia (CFP), que se incrementou e tornou-se o meio mais usual nesse contexto da pandemia de COVID-19. Houve um aumento considerável de demandas de saúde mental e da busca por esse tipo de atendimento, principalmente, em função do isolamento social e das diferentes modalidades de sofrimento psíquico desencadeadas pela pandemia. A expansão do atendimento psicológico on-line não se deu apenas entre os profissionais que trabalham em consultórios ou em clínicas particulares. Segundo o autor, esse aumento de demanda foi acompanhado de iniciativas importantes de implementação de plataformas de atendimento pelo setor público, disponibilizando para a população e também para profissionais de saúde o acesso à orientação e atendimento a distância no contexto da COVID-19.

Dados recentes de pesquisas mostram que o atendimento psicológico on-line possibilita uma assistência qualificada diante de situações de luto pela morte de familiares, colegas ou amigos. Mas também, por meio desse tipo de tratamento, pode-se oferecer suporte e acolhimento no âmbito da saúde mental aos profissionais de saúde da linha de frente de enfrentamento da pandemia da COVID-19, além do fornecimento de orientações de saúde mental para a população em geral (Viana, 2020; Enumo, \& Linhares, 2020; Pacheco, \& Silva, 2020; Salek, \& Moser, 2020).

Nesse cenário, o atendimento psicológico online oferecido pela Secretaria Municipal de Saúde de Guaratinguetá teve como objetivo atender pessoas e trabalhadores de saúde que 
manifestassem dificuldades mais intensas, como sentimentos de solidão, tristeza ou abatimento. Também medos intensos, angústia, dificuldades de dormir, de permanecer em casa, ou ansiedade, além dos possíveis conflitos sujeitos a ocorrer com a intensificação da convivência familiar diária. Assim, buscou-se oferecer, a quem procurasse o serviço, atendimentos em consultas terapêuticas (Winnicott, 1984), ou em psicoterapia breve (Lemgruber, 1984) e com objetivos limitados, a partir da escuta e continência à pessoa atendida.

É importante sinalizar que, de acordo com Lescovar (2004), as consultas terapêuticas foram criadas pelo psicanalista inglês D. W. Winnicott, a partir da otimização das entrevistas clínicas. Nesse tipo de intervenção que consta de poucas sessões (de 3 a 4 consultas) desenvolve-se um espaço propício à ajuda psicológica e seus correspondentes fins terapêuticos. Por intermédio da interação cliente-terapeuta, alicerça-se comunicações entre os envolvidos a partir da mutualidade da experiência, possibilitando o aparecimento de necessidades e questões psíquicas do paciente que, a partir da escuta especializada do profissional, podem ser acolhidas, sustentadas e ressignificadas.

Já em relação à psicoterapia breve, também conhecida como psicoterapia de tempo limitado, que geralmente inclui entre 8 a 12 sessões, é um processo terapêutico focal, aplicado principalmente a situações emergenciais. Segundo Simon (1991), o objetivo da intervenção, nessas situações, é melhorar a eficácia adaptativa do sujeito, tornando o período de crise uma oportunidade de crescimento e ajudando o paciente a encontrar soluções adequadas para ela.

Então, fundamentando-se nesses enquadres psicoterapêuticos abreviados on-line, procurou-se oferecer um espaço de escuta à população de Guaratinguetá e aos profissionais de saúde, estimulando a busca de recursos para enfrentar dificuldades na situação de pandemia da COVID-19, e, com isso, minorar o sofrimento e favorecer uma melhor qualidade de vida.

\section{MATERIAIS E MÉTODOS}

A partir de reuniões realizadas na Secretaria Municipal de Saúde de Guaratinguetá/SP foi organizado um fluxograma para o atendimento psicológico on-line para pessoas que, em decorrência do sofrimento mental causado pela COVID-19 e o isolamento social associado a ela, demandassem esse tipo de tratamento. Como procedimento, o interessado deveria ligar para o Centro de Saúde do município, preencher uma ficha de identificação realizada pela equipe de enfermagem que, por sua vez, encaminhava o caso para o grupo de 17 psicólogos associados ao serviço (05 servidores municipais e 12 voluntários).

De acordo com a agenda e horário disponíveis de cada profissional, esse entrava em contato telefônico ou por outro meio eletrônico de comunicação com a pessoa a ser atendida para fazer o agendamento da primeira consulta e do tratamento posterior. Cabe ressaltar que o trabalho 
oferecido cumpriu as normas do sigilo profissional na acolhida das diferentes demandas individuais, de acordo com as recomendações do Código de Ética Profissional dos Psicólogos (CFP, 2014).

Foi também organizado um cronograma quinzenal de reuniões entre a equipe de profissionais, visando ao acompanhamento dos casos e a organização do serviço. Essas reuniões tiveram em média a duração de duas horas, e foram realizadas de forma remota, durante o período de abril a novembro de 2020. Ao final, todos os dados sociodemográficos dos participantes e da demanda pelo atendimento psicológico foram lançados em planilha de Excel e realizado o tratamento estatístico com análise descritiva dos dados de levantamento de frequência simples e relativa.

\section{RESULTADOS E DISCUSSÃO}

Ao longo do período de atendimento, ou seja, de abril a novembro de 2020 , foram atendidos de forma remota (on-line) 145 pessoas, perfazendo um total de 778 sessões, com média de 5,4 consultas por usuário, o que se mostrou adequado de acordo com a proposta de uma intervenção breve, focada na queixa mais urgente de cada um (Winnicott, 1984; Lemgruber, 1984). O atendimento por meio das TICs também se revelou eficaz, tal como mostram as pesquisas nesse campo ((Viana, 2020; Enumo, \& Linhares, 2020; Pacheco, \& Silva, 2020; Salek, \& Moser, 2020). A Tabela 1 apresenta os dados referentes à caracterização dos participantes e das demandas pelo atendimento psicológico on-line.

Tabela 1 - Características sociodemográficas e demandas para o Serviço de atendimento psicológico on-line de Guaratinguetá/SP

\begin{tabular}{lll}
\hline CARACTERÍSTICAS & $\mathbf{N}$ & $\%$ \\
\hline Gênero & & \\
Feminino & 115 & 79,3 \\
Masculino & 30 & 20,7 \\
Idade & & \\
$03-11^{3}$ & 06 & 04,1 \\
$12-18^{4}$ & 16 & 11,0 \\
$19-30$ & 18 & 12,4 \\
$31-40$ & 22 & 15,2 \\
$41-59$ & 51 & 35,2 \\
\hline
\end{tabular}

\footnotetext{
${ }^{3}$ Os casos de crianças foram atendidos por meio da orientação dos pais.

${ }^{4}$ Os adolescentes foram atendidos com o consentimento de seus pais, que também receberam orientações sobre o acompanhamento psicológico.
}

Rev. Psicol Saúde e Debate. Fev., 2021:7(1): 94-106. 


\begin{tabular}{|c|c|c|}
\hline $60-80$ & 32 & 22,1 \\
\hline \multicolumn{3}{|l|}{ Escolaridade } \\
\hline Analfabeto & 03 & 02,0 \\
\hline Fundamental Comp. & 24 & 16,5 \\
\hline Fundamental Incomp. & 23 & 15,9 \\
\hline Ensino Médio Comp. & 55 & 38,0 \\
\hline Ensino Médio Incomp. & 11 & 07,6 \\
\hline Superior Comp. & 16 & 11,0 \\
\hline Superior Incomp. & 12 & 08,3 \\
\hline Pós Graduação & 01 & 00,7 \\
\hline \multicolumn{3}{|l|}{ Profissão } \\
\hline Comércio & 17 & 11,7 \\
\hline Saúde & 10 & 06,9 \\
\hline Educação & 07 & 04,8 \\
\hline Do lar & 39 & 26,9 \\
\hline Desempregado & 24 & 16,6 \\
\hline Estudante & 23 & 15,9 \\
\hline Outros & 25 & 17,2 \\
\hline \multicolumn{3}{|c|}{ Queixa/Demanda por Atendimento ${ }^{5}$} \\
\hline Ansiedade & 70 & 45,4 \\
\hline Depressão & 29 & 18,9 \\
\hline Dificuldade Isolamento Social & 32 & 20,8 \\
\hline Luto & 08 & 05,2 \\
\hline Teste positivo para COVID-19 & 02 & 01,3 \\
\hline Conflitos Familiares & 13 & 08,4 \\
\hline
\end{tabular}

A respeito do gênero dos indivíduos atendidos pelo serviço, verificou-se que a grande maioria foi do sexo feminino, ou seja, 115 casos, representando $79,3 \%$ da demanda. Esses resultados vêm corroborar os achados de investigações que apontam as diferenças entre os gêneros masculino e feminino quanto aos cuidados com a saúde (Botton, Cúnico, \& Strey, 2017; Alves, 2011).

As mulheres são consideradas mais cuidadosas e os homens mais vulneráveis devido aos aspectos psicossociais, como: o machismo, as dificuldades em assumir estar doente e a dificuldade à acessibilidade aos serviços de saúde (Alves, 2011). Se em outras morbidades a busca pelo serviço de saúde pelas pessoas do sexo masculino é menor, o mesmo parece acontecer com a

\footnotetext{
${ }^{5} \mathrm{O}$ número de motivos para o atendimento psicológico on-line é maior que a quantidade de pessoas atendidas, pois houve pacientes que apresentaram mais de uma queixa.
}

Rev. Psicol Saúde e Debate. Fev., 2021:7(1): 94-106. 
pandemia da COVID-19 e suas repercussões, principalmente, em termos dos agravos à saúde mental.

No que se refere à idade, os resultados mostraram que, de forma geral, as pessoas mais velhas foram as mais motivadas a buscar ajuda psicológica profissional para as diferentes formas de sofrimento mental decorrente da pandemia da COVID-19 no município de Guaratinguetá. Mesmo sabendo que essa doença afeta pessoas de diferentes idades, esta é associada a uma mortalidade maior em pessoas mais velhas e em idosos. Diante disso, o isolamento social pregado pela Organização Mundial da Saúde (OMS) tem causado influências no comportamento dessa população, contribuindo para o desenvolvimento de diferentes modalidades de sofrimento psíquico, inclusive o medo da morte por comorbidades já existentes, especialmente, na população com mais idade (Almeida Costa, 2020). Talvez seja essa a explicação para a maior busca de ajuda psicológica entre os adultos de meia idade e os idosos nesse município.

Em se tratando da escolaridade, verificou-se que grande parte da clientela indicou ter ensino médio completo $(38,0 \%)$, ou ensino médio incompleto $(07,6 \%)$, e tais dados disponíveis parecem corroborar achados da literatura que indicam um aumento da escolaridade da população em geral, conforme apontado por Arcary (2017). O autor destaca que nos últimos 30 anos, a escolaridade média aumentou entre os brasileiros, principalmente para a população entre os 15 e 30 anos. Mas, segundo ele, os mais velhos ainda pesam muito, estatisticamente, e tiveram muito pouco acesso à educação, colocando-nos diante do problema da desvalorização da educação, com taxas elevadíssimas de evasão no Ensino Médio e Superior. A partir da análise dos dados de escolaridade dos participantes do serviço de atendimento psicológico on-line de Guaratinguetá, o referido município parece refletir a mesma realidade do contexto nacional mais abrangente, destacado por Arcary (2017), pois em sua grande maioria os atendidos eram adultos de meia idade (40-59 anos) e idosos (60-80 anos).

Sobre a profissão das pessoas que buscaram o tratamento psicológico on-line oferecido pela Secretaria Municipal de Saúde de Guaratinguetá, observou-se que uma parcela considerável dos atendidos por esse serviço ou era do lar (dona de casa), (26,9\%), ou não trabalhava (vários aposentados) ou estava desempregada (16,6\%), representado quase a metade do total de participantes. Esse dado pode ser entendido pelo fato de que, nessa amostra da população, muitos eram mais velhos ou idosos que, de acordo com Almeida Costa (2020), são os mais vulneráveis diante da pandemia da COVID-19 por conta das comorbidades e da idade avançada. E, considerando o risco real de contágio e de desenvolver a doença que pode avançar para um quadro grave, sentem-se ameaçados, desenvolvendo, assim, diferentes tipos de sofrimento psíquico que demandam ajuda profissional especializada.

Por outro lado, é notável a baixa aderência de profissionais de saúde ao serviço, e esse dado é bastante preocupante, pois de acordo com Teixeira et al (2020), um dos principais 
problemas, em se tratando desses profissionais que trabalham na linha de frente do enfrentamento da COVID-19 é o risco de contaminação que tem gerado afastamento do trabalho, doença e morte, além de intenso sofrimento psíquico, que se expressa em transtorno de ansiedade generalizada, distúrbios do sono, medo de adoecer e de contaminar colegas e familiares. Portanto, na possibilidade de continuidade desse serviço de escuta psicológica on-line, deve-se intensificar a divulgação do trabalho, principalmente, nas diferentes unidades de saúde do município.

Ao analisar a queixa ou a demanda para o atendimento on-line, constatou-se que a ampla maioria referiu quadros psicopatológicos mais graves, especialmente, os transtornos de ansiedade e de depressão. Esses resultados corroboram os achados de outras pesquisas na área que destacam que durante o período da pandemia de COVID-19 no Brasil ascenderam, em muito, os casos de depressão e de ansiedade, com prevalências mais elevadas em adultos, mulheres e, especialmente, pessoas com diagnóstico prévio de depressão ou de transtornos de ansiedade generalizados (Barros et al., 2020). Diante desse resultado, e entendendo que a pandemia pode se estender em função do aumento do número de casos e de mortes, em uma segunda onda de contágio da doença e por causa das dificuldades de elaboração de um plano nacional de imunização até o momento, torna-se fundamental que o tratamento psicológico especializado, mesmo que de forma remota, seja oferecido para a população geral e também para os profissionais de saúde.

Destacou-se também o número de pessoas que apresentavam dificuldades em manter o isolamento social preconizado pela OMS. Segundo Dugnani (2020), a mudança de ritmo imposta pelo COVID-19 desencadeou a desaceleração repentina da velocidade de produção e de performance social, levando as pessoas a sentirem um impacto importante produzido pelo efeito da inércia, criada pela frenagem que as medidas tomadas para conter o avanço da doença promoveram. Com essa desaceleração, muitos indivíduos perderam o sentido de vida, se vendo obrigados a viver essa nova normalidade a qual não estavam acostumados. O enfrentamento dessa realidade de distanciamento e/ou isolamento social não foi fácil e, em função disso, tais pessoas demandaram uma escuta especializada para compartilhar suas dificuldades.

Embora com um número mais reduzido, foram registrados casos de conflitos familiares, incluindo episódios de violência doméstica e até mesmo de abuso sexual. Segundo Waksman e Blank (2020), a violência doméstica disparou no mundo diante da pandemia de COVID-19, principalmente pelo isolamento social e, consequentemente, pelo contato interpessoal intensificado entre os membros da família. O autor destaca a importância de disponibilizar serviços de apoio, se as vítimas não conseguem denunciar. Também sinaliza a necessidade da oferta de serviços e aplicativos para registrar queixas, processamento rápido das denúncias, aumentar 0 compartilhamento de informações sobre equipamentos de referência para a mulher e/ou crianças expostas à violência doméstica, reforçar campanhas publicitárias para conscientizar pessoas a denunciar, prestar assistência financeira às famílias necessitadas, incentivar projetos de apoio a 
vulneráveis em situações de vitimização, com base nos atendimentos médico, psicológico, de assistência social e jurídico. E, pontua que os profissionais da saúde exercem papel importante para a identificação e notificação das diferentes modalidades de violência doméstica.

Outro dado que chamou a atenção foi que apenas dois pacientes com teste positivo para COVID-19 buscaram o serviço de atendimento psicológico on-line. Considerando que o serviço foi pensado e planejado também para essa população, e que os pacientes acometidos por essa doença padecem de intenso sofrimento psíquico, que se expressa em transtorno de ansiedade generalizada, medo de morrer e de contaminar familiares e outras pessoas (Ornell, 2020), é fundamental o acolhimento de suas angústias para que possam enfrentar de forma mais adequada o tratamento de saúde. Por outro lado, a baixíssima adesão ao serviço de apoio psicológico on-line por esses pacientes no município de Guaratinguetá pode indicar que a divulgação do trabalho não foi realizada de forma eficaz. Mais uma vez, destaca-se a importância de que, numa possível continuidade do trabalho, uma ampla comunicação deve ser feita nos diferentes equipamentos de saúde e junto a todos profissionais da área para que seja possível acolher e apoiar os pacientes com teste positivo ou já em tratamento da COVID-19.

\section{CONSIDERAÇÕES FINAIS}

Verificou-se que o trabalho de apoio psicológico on-line disponibilizado pela Secretaria Municipal de Saúde para a população de Guaratinguetá cumpriu de forma satisfatória os objetivos propostos. Todos que se inscreveram no projeto foram acolhidos, atendidos e tratados de acordo com a modalidade de Consultas Terapêuticas e/ou Psicoterapia Breve, dando atenção à queixa mais urgente de cada caso. Aqueles que demandaram um tratamento mais longo ou que padeciam de algum transtorno psíquico mais grave foram encaminhados para outros serviços da Secretaria, especialmente para os equipamentos de Saúde Mental.

Porém, cabe destacar que foram identificados alguns entraves para o pronto encaminhamento de pacientes a esses serviços, mais especificamente para agendamento de consulta com o psiquiatra. Em função do exposto, sugere-se uma reformulação do fluxo de funcionamento no âmbito do Ambulatório de Saúde Mental e dos Centros de Atenção Psicossocial (CAPS), visto que os profissionais de psicologia se veem impedidos de realizar encaminhamentos diretos para consultas psiquiátricas, isso porque todo paciente deve, nesse fluxograma, agendar primeiro uma consulta com o clínico geral da UBS de referência, que encaminha o caso para o Ambulatório de Saúde Mental ou para o CAPS. Entendendo que o(a) psicólogo(a) possui as competências necessárias para identificar as demandas de acompanhamento psiquiátrico, sugerese que este(a) especialista possa realizar tais encaminhamentos sem atravessamento de um 
profissional de medicina que, muitas vezes, não tem o mesmo preparo e formação no campo da Saúde Mental.

Como já sinalizado, é importante que o trabalho de tratamento psicológico on-line tenha continuidade em função do aumento de casos e de mortes pela COVID-19, o que poderá desencadear maiores agravos na saúde mental da população. Contudo, é necessária ampliação do quadro de psicólogos concursados pela Secretaria de Saúde ou de contratação temporária de outros profissionais para dar conta da possível demanda a esse serviço especializado.

Também é aconselhado que haja uma ampla divulgação do trabalho, dando uma atenção maior para as unidades de saúde e aos diferentes profissionais da rede. Da mesma forma, orientase que seja estabelecida uma interlocução entre as diferentes secretarias da Prefeitura Municipal de Guaratinguetá, principalmente, envolvendo Saúde, Educação e Assistência Social, pois a saúde mental envolve vários fatores e determinantes que demandam, sempre, uma ação multiprofissional, transdisciplinar e interinstitucional para a melhor condução, encaminhamento e tratamento dos casos.

\section{REFERÊNCIAS}

Almeida Costa, F., Santos Silva, A., Oliveira, C. B. S., Costa, L. C. S., Silva Paixão, M. É., Celestino, M. N. S., ... \& de Lima Santos, I. L. V. (2020). COVID-19: seus impactos clínicos e psicológicos na população idosa. Brazilian Journal of Development, 6(7), 49811-49824. Recuperado de https://www.brazilianjournals.com/index.php/BRJD/article/view/13704

Alves, R. F., Silva, R. P., Ernesto, M. V., Lima, A. G. B., \& Souza, F. M. (2011). Gênero e saúde: o cuidar do homem em debate. Psicologia: teoria e prática, 13(3), 152-166. Recuperado de https://www.redalyc.org/pdf/1938/193821358012.pdf

Arcary, V. (2017). Um Brasil menos desigual? Mobilidade social baixa e evolução lenta da escolaridade média. Revista@mbienteeducação, 3(1), 9-17. Recuperado de http://publicacoes.unicid.edu.br/index.php/ambienteeducacao/article/view/164

Barros, M. B. D. A., Lima, M. G., Malta, D. C., Szwarcwald, C. L., Azevedo, R. C. S. D., Romero, D., ... \& Gomes, C. S. (2020). Relato de tristeza/depressão, nervosismo/ansiedade e problemas de sono na população adulta brasileira durante a pandemia de COVID-19. Epidemiologia e Serviços de Saúde, 29, e2020427. doi: 10.1590/S1679-49742020000400018

Botton, A., Cúnico, S. D., \& Strey, M. N. (2017). Diferenças de gênero no acesso aos serviços de saúde: problematizações necessárias. Mudanças-Psicologia da Saúde, 25(1), 67-72. Recuperado de metodista/index.php/MUD/article/view/7009

https://www.metodista.br/revistas/revistas-

Conselho Federal de Psicologia. (2014). Código de ética profissional do psicólogo. Brasília: CFP.

Dal'Bosco, E. B., Floriano, L. S. M., Skupien, S. V., Arcaro, G., Martins, A. R., \& Anselmo, A. C. C. (2020). A saúde mental da enfermagem no enfrentamento da COVID-19 em um hospital universitário regional. Revista Brasileira de Enfermagem, 73. doi: 10.1590/0034-7167-20200434

Rev. Psicol Saúde e Debate. Fev., 2021:7(1): 94-106. 
Dugnani, P. (2020). Hipermodernidade e a desaceleração do ritmo de vida provocada pela pandemia de COVID-19: Hypermodernity and the slowdown in the pace of life caused by the COVID-19 pandemic. Comunicação \& Inovação, 21(47). Recuperado de https://seer.uscs.edu.br/index.php/revista comunicacao inovacao/article/view/7117

Enumo, S. R. F., \& Linhares, M. B. M. (2020). Contribuições da Psicologia no contexto da Pandemia da COVID-19: seção temática. Estudos de Psicologia (Campinas), 37. doi: 10.1590/1982$0275202037200110 \mathrm{e}$

Instituto Brasileiro de Geografia e Estatística (IBGE) (2017). Base de dados por municípios das Regiões Geográficas Imediatas e Intermediárias do Brasil. Rio de Janeiro: IBGE. Recuperado em 29 de dezembro de 2020, de www.ibge.gov.br

Lemgruber, V. B. (1984). Psicoterapia breve a técnica focal. Porto Alegre, RS: Artes Médicas.

Lescovar, G. Z. (2004). As consultas terapêuticas e a psicanálise de D. W. Winnicott. Estudos de Psicologia (Campinas), 21(2), 43-61. doi: 10.1590/S0103-166X2004000200004

Ministério da Saúde (2020). Painel Coronavírus. Brasília: Ministério da Saúde. Recuperado em 29 de dezembro de 2020, de https://covid.saude.gov.br

Ornell, F. E. L. I. P. E., Schuch, J. B., Sordi, A. O., \& Kessler, F. H. P. (2020). Pandemia de medo e COVID-19: impacto na saúde mental e possíveis estratégicas. Revista debates in psychiatry. Recuperado de PandemiademedoeCOVID-19impactona20200506-102677-146aa84.pdf

Pacheco, T. R., \& Silva, D. M. D. (2020). Cuidado psicológico on-line ofertado no contexto de pandemia pelo Programa Acolher da Universidade do Extremo Sul Catarinense. Interfaces da COVID-19: impressões multifacetadas do período de pandemia, 80-81. doi: 10.18616/intcov

Salek, L., \& Moser, A. M. (2020). Atendimento psicológico on-line. Revista Científica Sophia, 1(1), 99-110. Recuperado de http://ojs.avantis.edu.br/index.php/sophia/article/view/111

Schmidt, B., Crepaldi, M. A., Bolze, S. D. A., Neiva-Silva, L., \& Demenech, L. M. (2020). Saúde mental e intervenções psicológicas diante da pandemia do novo coronavírus (COVID-19). Estudos de Psicologia (Campinas), 37, e200063. doi: 10.1590/1982-0275202037e200063

Simon, R. (1991). Psicanálise e psicoterapia breve. Psicologia/USP, 5 (1), 93-96.

Teixeira, C. F. D. S., Soares, C. M., Souza, E. A., Lisboa, E. S., Pinto, I. C. D. M., Andrade, L. R. D., \& Espiridião, M. A. (2020). A saúde dos profissionais de saúde no enfrentamento da pandemia de COVID-19. Ciência \& Saúde Coletiva, 25, 3465-3474. doi: 10.1590/141381232020259.19562020

Viana, D. M. (2020). Atendimento psicológico online no contexto da pandemia de COVID-19. Cadernos ESP-Revista Científica da Escola de Saúde Pública do Ceará, 14(1), 74-79. Recuperado de https://cadernos.esp.ce.gov.br/index.php/cadernos/article/view/399

Waksman, R. D., \& Blank, D. A importância da violência doméstica em tempos de COVID-19. Revista Residência Pediátrica. Recuperado de https://cdn.publisher.gn1.link/residenciapediatrica.com.br/pdf/rp240920a03.pdf

Winnicott, D. W. (1984). Consultas terapêuticas em Psiquiatria Infantil. Rio de Janeiro: Imago. 
World Health Organization (WHO). (2002). Relatório sobre a saúde no mundo: Saúde mental: nova concepção, nova esperança. Lisboa: WHO.

World Health Organization (WHO). (2020). Coronavirus disease (COVID-19) pandemic. Genebra: WHO. Recuperado em 29 de dezembro de 2020, de https://www.who.int/emergencies/diseases/novel-coronavirus-2019 